(c) 2019 Universidad Nacional Autónoma de México, Facultad de Estudios Superiores Zaragoza.

Este es un artículo Open Access bajo la licencia CC BY-NC-ND (http://creativecommons.org/licenses/by-nc-nd/4.0/).

TIP Revista Especializada en Ciencias Químico-Biológicas, 22: 1-13, 2019.

DOI: 10.22201/fesz.23958723e.2019.0.179

\title{
Identificación de compuestos fenólicos en extractos de almendra (Prunus dulcis) y nuez pecana (Carya illinoinensis) mediante cromatografía líquida acoplada a espectrometría de masas en tándem (HPLC-MS/MS)
}

\author{
Laura A. de la Rosa ${ }^{1}$, Emilio Álvarez-Parrilla ${ }^{1}$ y Jorge A. García-Fajardo² \\ ${ }^{1}$ Departamento de Ciencias Químico Biológicas, Instituto de Ciencias Biomédicas, Universidad \\ Autónoma de Ciudad Juárez, Anillo Envolvente del Pronaf y Estocolmo s/n, Ciudad Juárez, Chihuahua \\ 32310, México. ${ }^{2}$ CIATEJ (Centro de Investigación y Asistencia en Tecnología y Diseño del Estado de \\ Jalisco) Unidad Noreste, Apodaca, Nuevo León, México. E-mail: ${ }^{1}$ ldelaros@uacj.mx
}

\begin{abstract}
RESUMEN
La almendra y nuez pecana son alimentos funcionales, cuyo consumo habitual puede prevenir el desarrollo de numerosas enfermedades crónico-degenerativas. Los compuestos fenólicos (CF) son algunos de los que poseen mayor actividad biológica en estos frutos secos, pero su identificación y caracterización siempre representa un reto analítico. El objetivo del presente trabajo fue caracterizar el perfil de los CF, mediante HPLC acoplado a espectrometría de masas en tándem (MS/ MS) de dos tipos de extractos de nuez pecana y almendra, un extracto etanólico y uno acetónico. Se identificaron, mediante HPLC acoplado a espectrometría de masas de alta resolución (Q-TOF), 29 compuestos en almendra (22 estuvieron en el extracto acetónico y 24 en el etanólico) y 43 en nuez pecana (39 en el acetónico y 37 en el etanólico). La identidad de 6 compuestos de la almendra y 20 de nuez pecana se confirmó mediante el análisis de sus patrones de fragmentación en el modo MS/MS del equipo. El perfil de los CF fue claramente diferente entre almendra y nuez, pero muy parecido entre ambos tipos de solventes empleados (acetona y etanol), para un mismo fruto seco. En la almendra predominó la presencia de flavonoles y flavanonas, mientras que en nuez pecana predominaron taninos hidrolizables (sobre todo elagitaninos y derivados simples del ácido elágico) y condensados (hasta tetrámeros). En este estudio se describe por primera vez la identificación de tres elagitaninos en nuez pecana.
\end{abstract}

Palabras Clave: alimentos funcionales, frutos oleaginosos, taninos condensados, taninos hidrolizables, flavonoides.

Identification of phenolic compounds in almond (Prunus dulcis) and pecan (Carya illinoinensis) extracts by liquid chromatography coupled to tandem mass spectrometry (HPLC-MS/MS)

\begin{abstract}
Almonds and pecans are considered functional foods because their regular intake provides protection against several chronic-degenerative diseases. Phenolic compounds are some of the most bioactive components of these tree nuts; yet, their identification and characterization is regularly viewed as an analytical challenge. The aim of the present work was to characterize the phenolic compound profile of acetone and methanol extracts of almond and pecan nut, by using HPLC coupled to tandem mass spectrometry (MS/MS). First, high resolution MS (Q-TOF) was used to identify phenolic compounds in the extracts: 29 compounds were detected in almond (22 in acetone extract, 24 in ethanol extract) and 43 in pecan (39 in acetone extract, 37 in ethanol extract). Identity of 6 almond compounds and 20 pecan compounds was confirmed through analysis of their MS/MS fragmentation patterns. Phenolic profiles were different between pecan and almond but similar between extraction solvents for a same tree nut species. Flavonols and flavanones were the major types of phenolic compounds in almond, while pecan phenolics were mostly hydrolysable (ellagitannins acid ellagic acid derivatives) and condensed tannins (upto tetramers). Three ellagitannins are described for the first time in pecan.
\end{abstract}

Key Words: functional foods, oleaginous fruits, condensed tannins, hydrolysable tannins, flavonoids.

Nota: Artículo recibido el 06 de agosto del 2018 y aceptado el 14 de junio del 2019. 


\section{INTRODUCCIÓN}

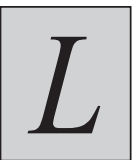

a almendra y nuez pecana son los frutos del Almendro (Prunus dulcis) y del Pecano (Carya illinoinensis) respectivamente. Son clasificadas como frutos secos, o frutos oleaginosos por su bajo contenido de agua y elevado contenido de aceite, en comparación con las frutas convencionales, por ejemplo, la manzana. Han sido consumidas como alimento por los seres humanos desde épocas muy remotas por su alto valor nutritivo (Alasalvar \& Shahidi, 2008). Hace algunas décadas, su consumo era poco recomendado por médicos y profesionales de la nutrición debido a su alto contenido lipídico, sin embargo, multitud de estudios han demostrado que el consumo habitual de frutos secos incluyendo nueces, almendras avellanas y cacahuates (alrededor de 30-40 g diarios), está asociado con una menor mortalidad por enfermedades del corazón y otros padecimientos como los crónico degenerativos, incluso se le relaciona con una mayor esperanza de vida (Aune et al., 2016); por lo que actualmente son recomendadas por diversas asociaciones americanas y europeas para la protección de la salud (Chang, Alasalvar, Bolling \& Shahidi, 2016).

Por su alto contenido de ácidos grasos insaturados y tocoferoles (formas de la vitamina E), las propiedades benéficas de las nueces se atribuyeron sobre todo a la porción lipídica de estos alimentos; sin embargo, los frutos secos también son ricos en antioxidantes polares (compuestos fenólicos, CF) y micronutrientes, por lo que es muy probable que el mecanismo de protección sea dependiente de los efectos sinérgicos entre los numerosos componentes bioactivos de estos alimentos (Chang, Alasalvar, Bolling \& Shahidi, 2016). Entre los frutos secos, las almendras destacan por su alto contenido de $\alpha$-tocoferol (la forma más bioactiva de la vitamina E) y son los frutos secos más producidos y consumidos a nivel mundial (Chang, Alasalvar, Bolling \& Shahidi., 2016). La nuez pecana posee el mayor contenido de CF y una muy elevada capacidad antioxidante. A nivel mundial su producción y consumo no son muy altos, estando en el $5^{\circ}$ y $6^{\circ}$ lugar entre los frutos secos, pero a nivel nacional, es un producto sumamente importante, ya que México es el primer productor y exportador (ÁlvarezParrilla, Urrea-López \& de la Rosa, 2018). Por su menor presencia mundial, la nuez pecana no ha sido tan estudiada como la almendra, sin embargo, se ha reportado que su perfil de CF es sumamente complejo, ya que es rico en taninos condensados e hidrolizables (Robbins, Ma, Wells, Greenspan \& Pegg, 2014). En cuanto a la identidad de los CF de la almendra, también contiene taninos condensados, pero es más rica en flavonoides monoméricos (Monagas, Garrido, LebrónAguilar, Bartolomé \& Gómez-Cordovés, 2007).

Varios trabajos que han caracterizado el perfil de los CF de nuez pecana y almendra demuestran que éste puede variar en respuesta a factores intrínsecos de la muestra, como por ejemplo: la variedad, región y condiciones de cultivo, tratamiento y almacenamiento poscosecha, así como por condiciones experimentales de extracción. Por otra parte, el uso cada vez más habitual de nuevas herramientas analíticas, como la cromatografía líquida acoplada a espectrometría de masas en tándem (HPLC-MS/MS) permite identificar un mayor número de compuestos con menor manipulación de los extractos, que da una idea más clara de las estructuras nativas de los compuestos presentes en las plantas silvestres y en especies vegetales cultivadas. Por ello, el objetivo del presente trabajo fue caracterizar el perfil de los CF, mediante HPLC-MS/MS de dos tipos de extractos de nuez pecana y almendra, un extracto etanólico y uno acetónico. El primero es el disolvente utilizado preferentemente para la extracción de los CF monoméricos, mientras que el segundo es utilizado para la extracción de compuestos poliméricos (de la Rosa, Álvarez-Parrilla \& Shahidi, 2011). En lo que respecta a un perfil fenólico detallado, se compara el efecto de la naturaleza del solvente frente al de las diferencias naturales entre dos diferentes especies de fruto seco. Asimismo, se identifica y se caracterizan los patrones de fragmentación de los compuestos bioactivos presentes en los extractos crudos de ambas especies con el objeto de profundizar en el conocimiento de este importante grupo de alimentos.

Los CF presentes en productos alimenticios son reconocidos por su actividad antioxidante, que puede tener efectos positivos en la conservación del alimento, así como en la salud del consumidor. Adicionalmente, se ha demostrado que los CF presentan múltiples actividades biológicas, incluyendo la regulación de la expresión de genes, la inhibición de enzimas y la modulación de la microbiota entérica (Sauceda et al., 2018; Lamuel-Raventos \& St. Onge, 2017). Por este motivo, es importante conocer el perfil y contenido de los $\mathrm{CF}$ en los alimentos que consumimos, sin embargo, la gran diversidad estructural de los mismos dificulta esta labor. Existen más de 8,000 CF en el reino vegetal, la mayoría de los cuales se pueden agrupar en las categorías de flavonoides y ácidos fenólicos. Sin embargo, éstos rara vez se encuentran en la naturaleza en forma de compuestos libres, sino que más bien se presentan glicosilados (generalmente unidos a mono o disacáridos) esterificados con ácidos orgánicos, o incluso ligados con macromoléculas o formando complejas estructuras poliméricas.

La identificación de los CF individuales, se ha realizado principalmente por técnicas de cromatografía líquida de alta eficiencia (HPLC); sin embargo, estos análisis se han visto limitados en gran parte por la falta de estándares de las formas nativas (glicosiladas, esterificadas, etc) de estos compuestos. Actualmente, el uso de detectores de masas acoplados a equipos de HPLC, aunado al desarrollo de técnicas más eficientes de cromatografía de ultra alta eficiencia (UPLC) ha permitido mejorar la capacidad de detección e identificación de un gran número de compuestos individuales en matrices 
altamente complejas, como lo son los alimentos y extractos fenólicos de alimentos (Motilva, Serra \& Macià, 2013). Los detectores de masas facilitan la identificación de señales cromatográficas por la asignación de un valor de masa/carga específico para cada una de ellas, incluso cuando las señales de dos o más compuestos coinciden; sin embargo, la identificación de compuestos nuevos, para los que no hay estándares disponibles, requiere del uso de equipos de masas en tándem que proveen información estructural gracias a los patrones de fragmentación de los iones precursores seleccionados (Abu-Reidah, 2013).

En el presente trabajo, se analizaron extractos de nuez pecana y almendra mediante un sistema de HPLC acoplado a masas en tándem (HPLC-QTOF), tomando en cuenta que las características de la columna y método cromatográfico se pueden considerar propias de una cromatografía de ultra alta eficiencia (Motilva, Serra \& Macià, 2013). Asimismo, el detector de tiempo de vuelo (TOF) tiene la capacidad de asignar masas con alta exactitud (errores menores a $10 \mathrm{ppm}$ ), permitiendo identificar compuestos diferentes con masas muy similares. Se comparó además la identidad de los compuestos presentes en extractos de acetona: agua con la de extractos etanol: agua y aunque ambos solventes tienen características similares, se ha reportado a la acetona como uno de los mejores solventes para extraer polifenoles poliméricos de tipo taninos, muy abundantes en las nueces (Álvarez-Parrilla, Urrea-López $\&$ de la Rosa, 2018); mientras que el etanol presenta ventajas por ser considerado apto para el consumo humano, pero hay muy pocos estudios que analizan extractos etanólicos de nueces por HPLC acoplado a masas. En todas las muestras analizadas se realizó una primera identificación (identificación tentativa) de CF mediante el software Qualitative analysis B.07.00 (Agilent Technologies, Palo Alto, CA, USA) utilizando un algoritmo que combina la asignación de masas exactas con la comparación de los patrones de distribución isotópica de los iones extraídos de cada señal cromatográfica.

\section{Materiales Y MÉTOdos}

\section{Extracción de CF}

Las muestras de almendra y nuez pecana fueron adquiridas en comercios de Ciudad Juárez en abril de 2017; las de nuez se descascararon manualmente desechando las cáscaras, las de almendra provenientes de Estados Unidos se compraron sin cáscara. Antes de extraer los CF se realizó un proceso de desgrasado, de acuerdo a la metodología reportada por de la Rosa, Álvarez-Parrilla \& Shahidi (2011). Para ello, se homogenizaron $10 \mathrm{~g}$ de nuez o almendra con 100 $\mathrm{mL}$ de hexano en un homogenizador (Thomas Scientific, Swedesboro, NJ, USA) a $8000 \mathrm{rpm}$ durante 3 minutos, la mezcla se filtró al vacío con papel filtro Whatman \# 4. Se separó el hexano conteniendo el aceite y el procedimiento se repitió dos veces más sobre el residuo sólido. Finalmente, el aceite se recuperó de los extractos hexánicos combinados, mientras que el residuo sólido (harina desgrasada) se secó al aire durante toda la noche en una campana de extracción y se almacenó a $-20{ }^{\circ} \mathrm{C}$ hasta su uso como fuente de CF. Los CF se obtuvieron mediante una extracción con solvente asistida por ultrasonido (Torres-Aguirre et al., 2018). Para ambas muestras se obtuvieron dos tipos de extractos: uno con etanol y otro con acetona ambos al $80 \% \mathrm{v} / \mathrm{v}$ en agua destilada. Un gramo de harina desgrasada se colocó en un tubo de $25 \mathrm{~mL}$, se agregaron $10 \mathrm{~mL}$ de solvente y se colocaron en un baño de ultrasonido (Branson modelo 5800, Danbury, CT, USA) durante 20 minutos a temperatura ambiente. A continuación, los tubos se centrifugaron en una centrífuga refrigerada (Eppendorff modelo AG 5810R, Hamburg, Germany) durante $15 \mathrm{~min}$ a $3,500 \mathrm{rpm}$ y a $4{ }^{\circ} \mathrm{C}$; se recuperó el sobrenadante y el precipitado fue re-extraído. Finalmente, los sobrenadantes de ambas extracciones fueron combinados, el solvente eliminado por medio de un rotavapor (Buchi modelo AG R-3, Flawil, Suiza) a $45^{\circ} \mathrm{C}$ y el agua restante por liofilización (Labconco Freezone 6, Kansas City, MO, USA) durante 48 h. Los extractos secos fueron empacados al vacío y almacenados hasta su análisis por HPLC-MS/MS.

\section{Identificación de $\mathbf{C F}$}

Los extractos secos fueron disueltos en metanol al $80 \%(\mathrm{v} / \mathrm{v}$ en agua) y diluidos (1:1) en agua para una concentración final de $2 \mathrm{mg}$ de extracto por $\mathrm{mL}$. Todos los disolventes fueron grado LC-MS. Estas muestras fueron filtradas a través de un filtro de jeringa de nylon, $0.45 \mu \mathrm{m}$ e inyectadas al equipo de HPLC acoplado a masas (HPLC-QTOF Agilent Modelo G6530, Agilent Technologies, Palo Alto, CA, USA) equipado con una columna Kinetex ${ }^{\circledR}$ biphenyl de 50 × $2.1 \mathrm{~mm}$ con un tamaño de partícula de $2.6 \mu \mathrm{m}$ y un tamaño de poro de $100 \AA$ (Phenomenex, Torrance, CA, USA) Para la separación cromatográfica se utilizó un gradiente de dos fases móviles (fase A, ácido fórmico al $0.1 \%$ en agua; fase $\mathrm{B}$, ácido fórmico al $0.1 \%$ en metanol) con un flujo de $0.4 \mathrm{~mL} / \mathrm{min}$ y un volumen de inyección de $1 \mu \mathrm{L}$. El gradiente se programó de la siguiente manera: $10-100 \%$ de B de los 0 a los 8 minutos; $100 \%$ B de los 8 a los 9.5 minutos; $100-10 \%$ de B de los 9.5 a los 9.6 minutos.

La detección e identificación de los CF se realizó mediante un analizador de masas en tándem cuadrupolo tiempo de vuelo (Q-TOF) con una fuente de ionización por electrospray (ESI). Se utilizó ionización negativa con las siguientes condiciones: voltaje del capilar 4,000 V, presión del gas nebulizador $40 \mathrm{psi}$, temperatura del gas de secado $350{ }^{\circ} \mathrm{C}$ y flujo de $10 \mathrm{~L} / \mathrm{min}$. Las condiciones de voltaje del TOF fueron: fragmentador $150 \mathrm{~V}$, skimmer $65 \mathrm{~V}$ y octopolo $750 \mathrm{~V}$. La fragmentación de los iones precursores se llevó a cabo mediante un método no dirigido (Auto MS/MS), seleccionando los 5 iones más abundantes de cada señal cromatográfica y una rampa de energías de colisión desde -10 hasta $-40 \mathrm{~V}$. Se utilizó un algoritmo de identificación por base de datos, donde la base de datos se 
construyó utilizando los CF más abundantes en alimentos, así como los CF reportados en la literatura característicos de diferentes tipos de frutos secos, limitándose a almendra y nuez pecana y otros de naturaleza química similar. Se realizó una identificación tentativa por comparación de la masa calculada vs la masa teórica (registrada en la base de datos) aunada al análisis de la distribución isotópica del ion extraído. Dentro de los criterios de identificación, también se consideró un porcentaje de identidad (score) mayor al $70 \%$, de acuerdo a las recomendaciones del fabricante, que recomienda un score mayor al $50 \%$ para la identificación tentativa, y una abundancia del ion mayor a 1,000 cuentas en los extractos de almendra y mayor a 5,000 cuentas en los extractos de nuez pecana debido a que la abundancia de los iones individuales fue más alta en general en los extractos de nuez pecana. La identidad de los compuestos más abundantes fue confirmada o descartada por su patrón de fragmentación el cual se comparó con el de bases de datos de espectros de masas (Metlin integrada al software MassHunter PCDL Manager for Metabolomics B.07.00 [Agilent Technologies, Palo Alto, CA, USA] y The Human Metabolome Database [www.hmdb.ca]) y lo reportado en literatura.

\section{RESULTADOS Y DISCUSIÓN}

En la Figura 1 se muestra, como ejemplo, el proceso para realizar la identificación tentativa y confirmación por MS/MS de la estructura de un compuesto abundante en los extractos de nuez pecana (monogaloil glucosa, compuesto 6 de la Tabla I). La figura $1 \mathrm{~A}$ corresponde al cromatograma de ion total (TIC). El primer análisis de la muestra consiste en obtener los cromatogramas de ion extraído (Figura 1B) de todos los compuestos cuyas masas exactas coincidían con las de la base de datos (con un error menor a 10 ppm). Para cada compuesto identificado se comparó la distribución isotópica encontrada en la muestra con la teórica de acuerdo con su fórmula molecular (Figura 1C). Con esta información se obtuvo la identificación tentativa en un primer nivel (Tablas II y I). A continuación se analizaron los patrones de fragmentación de los cinco iones más abundantes en cada tiempo de retención, en la Figura 1D se muestra el espectro de masas de monogaloil glucosa (rt 0.86), donde se observa la abundancia del ion precursor, de los fragmentos principales y las estructuras de los tres iones: el ion molecular (que fue el ion precursor) y los dos fragmentos más abundantes (estructuras teóricas según www.hmdb.ca).

La identificación tentativa de algunos compuestos indicó que, de acuerdo con la información de la base de datos, existe más de una posible correspondencia. Las correspondencias múltiples consistieron siempre en diversos isómeros con una misma masa molecular exacta, no en compuestos con masas moleculares similares, como es el caso de los compuestos glicosilados. Sin embargo, hay que considerar que dicha base de datos contiene información limitada, por lo que el número real de compuestos que pueden corresponder a una misma masa (incluso considerando la asignación con alta exactitud) y distribución isotópica determinadas es mucho mayor, ya que para, exactamente, la misma fórmula molecular predicha existen en algunos casos cientos de compuestos posibles (muchos de ellos ni siquiera son $\mathrm{CF}$ ). A pesar de que la identificación absoluta de un producto natural, mediante la elucidación de su estructura, requiere de un conjunto de técnicas analíticas, especialmente resonancia magnética nuclear, mediante el análisis de los patrones de fragmentación de sus iones moleculares en un espectrómetro de masas en tándem es posible hacer una predicción aproximada de su estructura; lo que, en conjunto con la masa exacta del ion molecular (el ion precursor), nos permite proponer su identidad con una baja incertidumbre (Abu-Reidah, 2013; Fabre, Rustan, Hoffmann \& Quetin-Leclercq, 2001; Justesen, 2000).

Una vez considerados todos los criterios de identificación descritos en la sección de Materiales y métodos, los compuestos así identificados en los extractos etanólicos y acetónicos de almendra y nuez pecana se muestran en las Tablas II y I.

Se encontraron un total de 29 compuestos en almendra (22 estuvieron presentes en el extracto acetónico y 24 en el etanólico) y 43 en nuez pecana igual que el anterior (39 en el acetónico y 37 en el etanólico).

En general, tanto el número de compuestos identificados como las abundancias de cada ion individual fueron mayores en los extractos de nuez pecana que en los de almendra. En cuanto a la comparación por solvente de extracción, la acetona resultó ligeramente más efectiva en cuanto al número de compuestos extraídos y la abundancia de los iones identificados; sin embargo, esta diferencia fue muy ligera y se puede atribuir a la diferencia en polaridad de ambos solventes. En ambas muestras se identificó ácido cítrico (en el extracto acetónico para la almendra y en el extracto etanólico para la nuez), siendo más abundante en la almendra. Hasta el momento, no se han encontrado reportes previos de la presencia de este compuesto en almendra o nuez pecana, quizá porque el presente estudio es uno de los primeros análisis no dirigidos que se realiza con estas nueces a diferencia de otros donde no se buscó la presencia de ácidos orgánicos. El número e identidad de los CF presentes en ambos frutos secos fueron, distintos, sin embargo, no hubo diferencias significativas por efecto del disolvente.

Los compuestos más abundantes en almendra fueron los flavonoides de las familias de los flavonoles y las flavanonas, mientras que en nuez pecana fueron taninos hidrolizables (sobre todo elagitaninos) y condensados (en el extracto acetónico) de bajo-mediano peso molecular y algunos ácidos hidroxibenzoicos. 

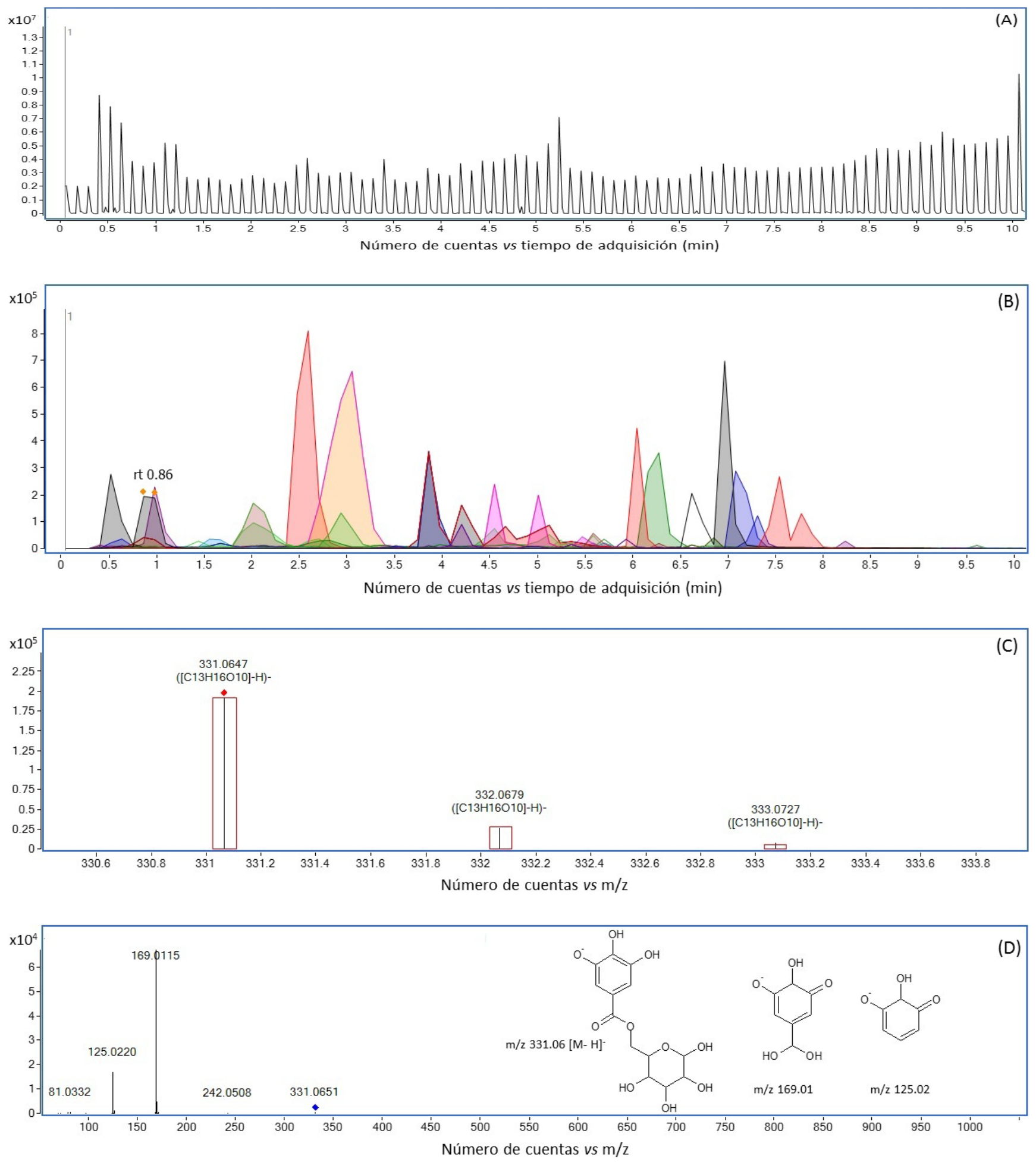

Figura 1. Identificación tentativa de la monogaloil-glucosa en un extracto etanólico de nuez pecana. (A) Cromatograma de ion total (TIC) en modo auto MS/MS del extracto. (B) Superposición de los cromatogramas de ion extraído (EIC) de los compuestos identificados tentativamente en el cromatograma mostrado en A. (C) Comparación de las distribuciones isotópicas identificadas para el ion 331.0647 en comparación con las teóricas de acuerdo a la fórmula de monogaloil glucosa. (D) Espectro de masas obtenido a partir del ion precursor 331.0651 (ion molecular de monogaloil glucosa), donde se muestran las estructuras predichas para el ion precursor y cada uno de sus fragmentos (www.hmdb.ca). Elaborada por los autores. 


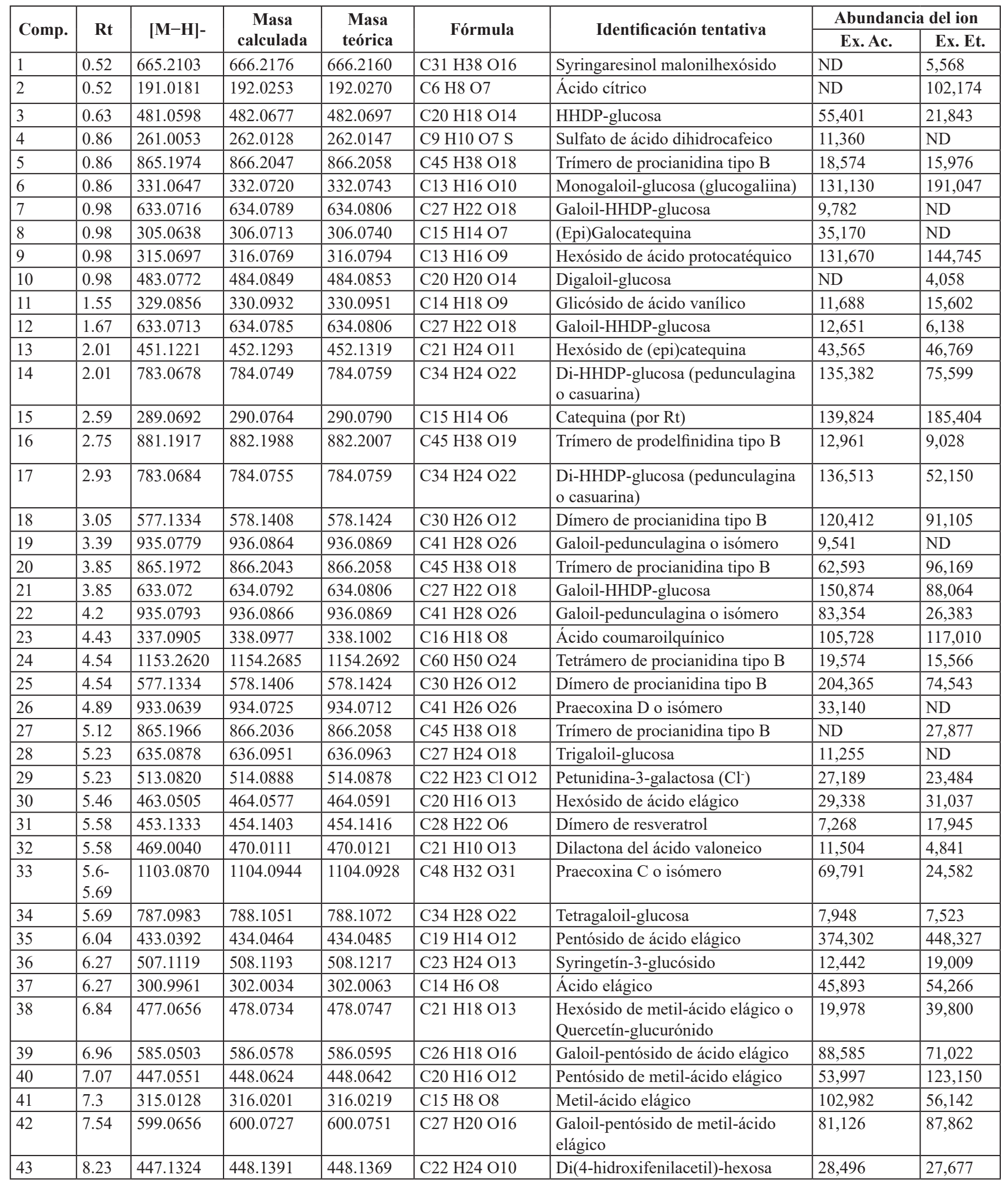

Rt: tiempo de retención; Ex. Ac: extracto acetónico 70\%; Ex. Et: extracto etanólico 70\%; HHDP: hexahidroxidifenoil. ND: no detectado.

Tabla I. Identificación tentativa de compuestos fenólicos en extractos de nuez pecana (Carya illinoinensis) mediante HPLC-MS. 


\begin{tabular}{|c|c|c|c|c|c|c|c|c|}
\hline \multirow{2}{*}{ Comp. } & \multirow{2}{*}{ Rt } & \multirow{2}{*}[\mathbf{M}-\mathbf{H}]{-} & \multirow{2}{*}{$\begin{array}{c}\text { Masa } \\
\text { calculada }\end{array}$} & \multirow{2}{*}{$\begin{array}{l}\text { Masa } \\
\text { teórica }\end{array}$} & \multirow{2}{*}{ Fórmula } & \multirow{2}{*}{ Identificación tentativa } & \multicolumn{2}{|c|}{ Abundancia del ion } \\
\hline & & & & & & & Ex. Ac. & Ex. Et. \\
\hline 1 & 0.51 & 191.0178 & 192.0251 & 192.0270 & C6 H8 O7 & Ácido cítrico & 212,251 & ND \\
\hline 2 & 0.63 & 665.2096 & 666.2169 & 666.2160 & C31 H38 O16 & Syringaresinol malonilhexósido & ND & 3,800 \\
\hline 3 & 0.86 & 147.0448 & 148.0520 & 148.0524 & C9 H8 O2 & Ácido cinámico & ND & 1,643 \\
\hline 4 & 0.97 & 315.0698 & 316.0776 & 316.0794 & C13 H16 O9 & Hexósido de ácido protocatéquico & 12,145 & ND \\
\hline 5 & 2.24 & 329.0867 & 330.0943 & 330.0951 & $\mathrm{C} 14 \mathrm{H} 18 \mathrm{O} 9$ & Glucósido de ácido vanílico & 6,831 & 4,599 \\
\hline 6 & 2.58 & 289.0698 & 290.0772 & 290.0790 & $\mathrm{C} 15 \mathrm{H} 14 \mathrm{O} 6$ & Catequina (por Rt) & 20,624 & 23,077 \\
\hline 7 & 3.62 & 465.1032 & 466.1105 & 466.1111 & $\mathrm{C} 21 \mathrm{H} 22 \mathrm{O} 12$ & Hexósido de taxifolina & ND & 1,894 \\
\hline 8 & 4.08 & 517.1535 & 518.1611 & 518.1636 & $\mathrm{C} 22 \mathrm{H} 30 \mathrm{O} 14$ & Dihexósido de ácido ferúlico & 9,997 & 10,670 \\
\hline 9 & 4.31 & 329.0859 & 330.0931 & 330.0951 & C14 H18 O9 & Glicósido de ácido vanílico & 10,414 & 7,071 \\
\hline 10 & 4.43 & 289.0696 & 290.0770 & 290.0790 & C15 H14 O6 & Epicatequina (por Rt) & 3,457 & 14,255 \\
\hline 11 & 5.00 & 577.1350 & 578.1427 & 578.1424 & $\mathrm{C} 30 \mathrm{H} 26 \mathrm{O} 12$ & Dímero de procianidina tipo B & 5,408 & 3,305 \\
\hline 12 & 5.92 & 499.0634 & 500.0713 & 500.0722 & $\mathrm{C} 21 \mathrm{H} 21 \mathrm{Cl} \mathrm{O} 12$ & Hexósido de delfinidina $\left(\mathrm{Cl}^{-}\right)$ & 8,086 & 4,666 \\
\hline 13 & 6.04 & 463.0863 & 464.0946 & 464.0955 & $\mathrm{C} 21 \mathrm{H} 20 \mathrm{O} 12$ & Hexósido de quercetina & 13,870 & 7,637 \\
\hline 14 & 6.15 & 287.0534 & 288.0607 & 288.0634 & C15 H12 O6 & Eriodictyol o Dihidrokaempferol & 57,302 & 82,012 \\
\hline 15 & 6.16 & 449.1064 & 450.1133 & 450.1162 & $\mathrm{C} 21 \mathrm{H} 22 \mathrm{O} 11$ & $\begin{array}{l}\text { Ramnósido de taxifolina o } \\
\text { Hexósido de eriodictyol o } \\
\text { dihidrokaempferol }\end{array}$ & ND & 6,335 \\
\hline 16 & 6.16 & 300.9976 & 302.0048 & 302.0063 & C14 H6 O8 & Ácido elágico & ND & 2,351 \\
\hline 17 & 6.38 & 593.1492 & 594.1567 & 594.1585 & $\mathrm{C} 27 \mathrm{H} 30 \mathrm{O} 15$ & \begin{tabular}{|l|} 
Diglicósido de apigenina o \\
Rutinósido de kaempferol
\end{tabular} & 40,491 & 23,488 \\
\hline 18 & 6.38 & 447.0908 & 448.0982 & 448.1006 & $\mathrm{C} 21 \mathrm{H} 20 \mathrm{O} 11$ & Hexósido de kaempferol & 19,502 & 4,736 \\
\hline 19 & 6.61 & 477.1021 & 478.1093 & 478.1111 & $\mathrm{C} 22 \mathrm{H} 22 \mathrm{O} 12$ & Glicósido de isoramnetina & 284,349 & 169,602 \\
\hline 20 & 6.73 & 433.1114 & 434.1191 & 434.1213 & $\mathrm{C} 21 \mathrm{H} 22 \mathrm{O} 10$ & Naringenina-C-hexósido & 34,759 & 21,561 \\
\hline 21 & 6.73 & 513.0769 & 514.0867 & 514.0878 & $\mathrm{C} 22 \mathrm{H} 23 \mathrm{ClO} 12$ & Petunidina-3-galactósido $\left(\mathrm{Cl}^{-}\right)$ & 5,105 & ND \\
\hline 22 & 6.96 & 301.0332 & 302.0401 & 302.0427 & $\mathrm{C} 15 \mathrm{H} 10 \mathrm{O} 7$ & Quercetina & 43,450 & ND \\
\hline 23 & 6.96 & 585.0509 & 586.0582 & 586.0595 & $\mathrm{C} 26 \mathrm{H} 18 \mathrm{O} 16$ & Ácido elágico-galoil-pentósido & ND & 4,722 \\
\hline 24 & 7.42 & 285.0377 & 286.045 & 286.0477 & C15 H10 O6 & Kaempferol o luteolina & 81,610 & 52,137 \\
\hline 25 & 7.42 & 271.0589 & 272.0661 & 272.0685 & $\mathrm{C} 15 \mathrm{H} 12 \mathrm{O} 5$ & Naringenina & 96,235 & 100,288 \\
\hline 26 & 7.65 & 315.0498 & 316.0569 & 316.0583 & C16 H12 O7 & Isoramnetina $\mathrm{o}$ azaleatina & 878,654 & 473,504 \\
\hline 27 & 7.65 & 301.0688 & 302.0762 & 302.0790 & C16 H14 O6 & Hesperetina & ND & 10,096 \\
\hline 28 & 8.22 & 447.1319 & 448.1388 & 448.1369 & $\mathrm{C} 22 \mathrm{H} 24 \mathrm{O} 10$ & Di(4-hidroxifenilacetil)-hexosa & 41,032 & 35,486 \\
\hline 29 & 8.57 & 329.0648 & 330.0720 & 330.0740 & C17 H14 O7 & Caryatina & 45,171 & ND \\
\hline
\end{tabular}

Rt: tiempo de retención; Ex. Ac: extracto acetónico 70\%; Ex. Et: extracto etanólico 70\%. ND: no detectado.

Tabla II. Identificación tentativa de compuestos fenólicos en extractos de almendra (Prunus dulcis) mediante HPLC-MS.

Sólo 7 compuestos identificados en la almendra se encontraron entre los iones que, por su abundancia, fueron fragmentados en sus respectivos tiempos de retención. Sus iones precursores y fragmentos se enlistan en la Tabla III, así como la confirmación o rectificación de su identificación tentativa (Tabla II), por comparación con los fragmentos reportados en bases de datos y literatura científica. La fragmentación del compuesto 1 (Rt 0.51, [M-H]- 191.0178) confirmó que se trata del ácido cítrico (el mismo compuesto con similar tiempo de retención se encontró también en nuez pecana), confirmado en la base de datos Metlin integrada al propio software de identificación. El compuesto 14 (Rt 6.15, [M-H]- 287.0534) mostró dos posibles coincidencias: eriodictyol (una flavanona) o dihidrokaempferol (un dihidroflavonol o flavanonol); ambos han sido reportados previamente en extractos de piel de almendra (Monagas, Garrido, Lebrón-Aguilar, Bartolomé \& Gómez-Cordovés, 2007), sin embargo, el patrón de fragmentación fue más parecido al de dihidrokaempferol, según lo reportado por Moqbel et al. (2018), quien indica que el fragmento de $\mathrm{m} / \mathrm{z}$ 259 corresponde a la pérdida de un grupo $\mathrm{CO}$, característica de flavanonoles, mientras que el fragmento de m/z 125 podría corresponder a la fragmentación del anillo A. Esta fragmentación del anillo $\mathrm{A}$ se ha reportado también en flavanonas (Fabre, Rustan, Hoffmann \& Quetin-Leclercq, 2001), sin embargo, la presencia de ambos fragmentos juntos coincide con lo observado por Moqbel et al. (2018) para dihidrokaempferol, al tiempo que no se encuentran ninguno de los fragmentos más característicos del eriodictyol, motivo por el cual se concluye que el compuesto 


\begin{tabular}{|l|l|l|l|l|l|}
\hline Comp. & $\begin{array}{c}\text { [M-H]- (ion } \\
\text { precursor }\end{array}$ & \multicolumn{1}{|c|}{ Fragmentos } & Fórmula & \multicolumn{1}{|c|}{$\begin{array}{c}\text { Identificación } \\
\text { por patrón de } \\
\text { fragmentación }\end{array}$} & $\begin{array}{l}\text { Referencia para comparación } \\
\text { de fragmentos }\end{array}$ \\
\hline 1 & 191.0178 & $111.007,87.008,85.028$ & C6 H8 O7 & Ácido cítrico & base de datos Metlin; \\
\hline 14 & 287.0534 & $259.058,125.022$ & C15 H12 O6 & Dihidrokaempferol & Moqbel et al., 2018 \\
\hline 17 & 593.1492 & 285.038 & C27 H30 O15 & $\begin{array}{l}\text { Rutinósido de } \\
\text { kaempferol }\end{array}$ & $\begin{array}{l}\text { Monagas, Garrido, Lebrón- } \\
\text { Aguilar, Bartolomé \& Gómez- } \\
\text { Cordovés, 2007 }\end{array}$ \\
\hline 19 & 477.1021 & $\begin{array}{l}314.041,271.021, \\
243.027,151.000\end{array}$ & C22 H22 O12 & $\begin{array}{l}\text { Glicósido de } \\
\text { isoramnetina }\end{array}$ & Moqbel et al., 2018 \\
\hline 25 & 271.0589 & $151.000,119.048$ & C15 H12 O5 & Naringenina & $\begin{array}{l}\text { base de datos Metlin; Fabre, } \\
\text { Rustan, Hoffmann \& Quetin- } \\
\text { Leclercq, 2001 }\end{array}$ \\
\hline 26 & 315.0498 & $300.025,151.000$ & C16 H12 O7 & Isoramnetina & HMDB; Justesen, 2000 \\
\hline 28 & 447.1319 & $\begin{array}{l}375.081,219.837, \\
162.836\end{array}$ & C22 H24 O10 & Desconocido & \\
\hline
\end{tabular}

Tabla III. Confirmación de la identificación tentativa de compuestos fenólicos en extractos de almendra, por comparación de sus patrones de fragmentación en modo negativa con el de bases de datos de espectros de masas (Metlin integrada al software MassHunter PCDL Manager for Metabolomics B.07.00 [Agilent Technologies, Palo Alto, CA, USA] y The Human Metabolome Database [www.hmdb.ca]) y lo reportado en la literatura científica.

14 corresponde a dihidrokaempferol. El compuesto 17 (Rt 6.38, [M-H]- 593.1492) mostró un fragmento de $\mathrm{m} / \mathrm{z} 285$, que corresponde a la masa del kaempferol como aglicona, mientras que la diferencia (308) corresponde a la pérdida de un rutinósido (disacárido glucosa-ramnosa), indicando la presencia del rutinósido de kaempferol, que ya ha sido reportado por Monagas, Garrido, Lebrón-Aguilar, Bartolomé \& Gómez-Cordovés, (2007) en piel de almendra. Aunque esta información no permite descartar la posibilidad de que la aglicona sea luteolina en lugar de kaempferol , (ya que ambos poseen la misma fórmula y masa molecular), no se ha reportado en almendra la presencia de rutinósido de luteolina, infiriendo con un alto grado de certidumbre que el compuesto 17 corresponde a un rutinósido de kaempferol. El compuesto 19 (Rt 6.61, [M-H]- 477.1021) se identificó como glucósido de isoramnetina, a pesar de que en un principio no presentó el ion característico de cualquier glicósido, que consiste en el ion de la aglicona $-\mathrm{H}$ (Justesen, 2000) que en este caso sería de una $\mathrm{m} / \mathrm{z} 315$. Sin embargo, los fragmentos con $\mathrm{m} / \mathrm{z}$ 314, 271 y 243 coinciden con los encontrados por Moqbel et al. (2018), quien identificó al glucósido de isoramnetina en hojas y tallos de árbol de almendra, con unas condiciones de ionización muy similares a las utilizadas en el presente trabajo; además el fragmento $\mathrm{m} / \mathrm{z} 151$ corresponde a un producto de fragmentación del anillo A característico de flavonas, flavonoles y flavanonas no metilados en dicho anillo, incluyendo a la isoramnetina (Fabre, Rustan, Hoffmann \& Quetin-Leclercq, 2001; Justesen, 2000). Los compuestos 25 (Rt 7.42, [M-H]- 271.0589) y 26 (Rt 7.65, [M-H]- 315.0498) fueron identificados fácilmente por tratarse de agliconas: naringenina (una flavanona) e isoramnetina (un flavonol), respectivamente. Sus patrones de fragmentación mostraron los fragmentos característicos descritos en bases de datos y referencias especializadas (Fabre, Rustan, Hoffmann \& Quetin-Leclercq, 2001; Justesen, 2000). Por último, el compuesto 28 (Rt 8.22, [M-H]- 447.1319) no pudo ser identificado. La identificación tentativa marcó un compuesto fenólico no flavonoide reportado en lechuga (Abu-Reidah, 2013) sin embargo, su patrón de fragmentación no coincidió.

En resumen, los CF más abundantes pudieron ser identificados y confirmada su estructura por HPLC-MS-MS en ambos extractos de almendra y fueron: 1) isoramnetina aglicona, 2) glicósido de isoramnetina y 3 ) naringenina aglicona. Los dos primeros son flavonoles metilados, y el último una flavanona.

En la Tabla IV se muestran los iones precursores y fragmentos obtenidos en el análisis de la nuez pecana. 24 iones precursores fueron fragmentados, de los cuales 3 se identificaron tentativamente como diferentes isómeros del mismo compuesto, dos procianidinas y un elagitanino. Los compuestos identificados por su patrón de fragmentación pueden dividirse en 5 grupos, según su naturaleza química: 1) glicósidos de ácidos fenólicos (compuesto 9); 2) flavan3-oles, incluyendo monómeros, glicósidos y procianidinas (compuestos 13, 15, 18, 20, 25 y 27); 3) galotaninos (compuestos 6 y 34$) ; 4$ ) derivados de ácido elágico, con o sin ácido gálico (compuestos 30, 35 y 37-42); y 5) elagitaninos, que contienen al menos una unidad de ácido hexahidroxidifénico (HHDP, compuestos 14, 17, 21, 22, 26 y 33). El compuesto 23 


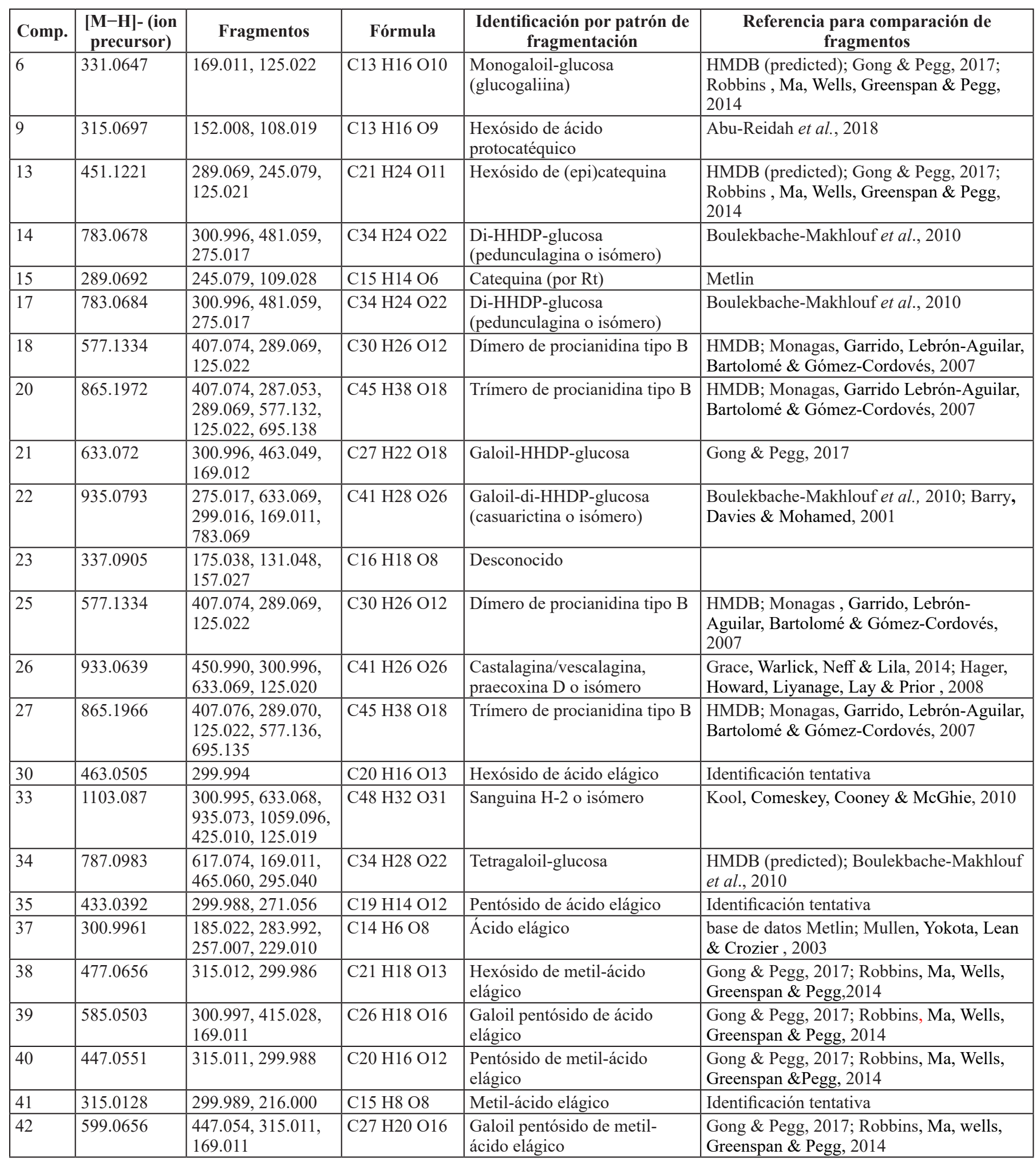

HHDP: hexahidroxidifenoil

Tabla IV. Confirmación de la identificación tentativa de compuestos fenólicos en extractos de nuez pecana, por comparación de sus patrones de fragmentación en modo negativa con el de bases de datos de espectros de masas (Metlin integrada al software MassHunter PCDL Manager for Metabolomics B.07.00 [Agilent Technologies, Palo Alto, CA, USA] y The Human Metabolome Database [www.hmdb.ca]) y lo reportado en la literatura científica. 
no pudo ser identificado, ya que su patrón de fragmentación no coincidió con el del ácido coumaroilquínico que había sido identificado tentativamente por su masa exacta y distribución isotópica (ver Tabla I).

El compuesto 9 (Rt 0.98, [M-H]- 315.0697) se identificó como un derivado glicosilado del ácido protocatéquico, aunque no presentó el típico fragmento correspondiente a la pérdida del residuo de azúcar (162) para dar un fragmento de $\mathrm{m} / \mathrm{z} 153$ ([M-H]- del ácido protocatéquico), los fragmentos con m/z 152 y 108 han sido detectados por Abu-Reidah, Ali-Shtayeh, Jamous, Arráez-Román \& Segura-Carretero, (2015) y corresponden también a la pérdida de un hidrógeno adicional, al residuo de azúcar, seguido de la pérdida de un $\mathrm{CO}_{2}$, característica de los ácidos fenólicos. Algunos hexósidos de ácido protocatéquico han sido reportados en extractos acetónicos de nuez pecana (Gong \& Pegg, 2017; Robbins, Ma, Wells, Greenspan \& Pegg, 2014).

Dentro de los flavan-3-oles, los compuestos 13 y 15 pudieron ser identificados como un hexósido de catequina o epicatequina y como catequina libre, respectivamente. La catequina ( $\mathrm{Rt}$ 2.59, [M-H]- 289.0692) pudo ser identificada fácilmente por su patrón de fragmentación comparable con el de la base de datos integrada al software, pero para poderla distinguir de su estereoisómero, la epicatequina, fue necesario comparar los Rt de estándares comerciales. En el caso del hexósido, se identificó claramente el fragmento correspondiente a la pérdida del residuo de azúcar (fragmento 289, correspondiente a [M-H]de catequina o epicatequina), así como otros productos de la fragmentación de la aglicona, aunque no fue posible confirmar de cuál de los dos estereoisómeros se trataba. Los compuestos 18 y 25 (Rt 3.05 y 4.54, [M-H]- 577.1334) corresponden a dímeros de procianidinas tipo $\mathrm{B}$, es decir, dímeros de (epi) catequina unidos entre sí mediante un solo enlace interflavan; mientras que los compuestos 20 y 27 (Rt 3.85 y 5.12, [M-H]$865.197)$ son trímeros. Sus patrones de fragmentación han sido descritos por Monagas, Garrido, Lebrón-Aguilar, Bartolomé \& Gómez-Cordovés, (2007): los fragmentos con m/z 695 en trímeros y 407 en dímeros y trímeros son producto de la fragmentación retro-Diels-Adler seguida de la eliminación de una molécula de agua; los fragmentos con m/z 289 en dímeros y trímeros y m/z 287 y 577 en trímeros corresponden a la ruptura del enlace interflavan por un mecanismo quinonametino (Monagas, Garrido, Lebrón-Aguilar, Bartolomé \& Gómez-Cordovés, 2007). Todos estos flavan-3-oles ya han sido reportados en extractos acetónicos de nuez pecana y en el presente trabajo mostramos también su presencia en el extracto etanólico; también se identificaron otros flavan-3-oles oligoméricos como un tetrámero de procianidina B (Rt 4.54, $[\mathrm{M}-\mathrm{H}]-1153.262)$ y un trímero de prodelfinidina B (Rt 2.75, [M-H]- 881.1917) que si bien no pudieron ser confirmados por su patrón de fragmentación, también han sido descritos como componentes importantes de la nuez pecana por varios autores (Álvarez-Parrilla, Urrea-López \& de la Rosa, 2018).

Los compuestos 6 y 34 se identificaron como galotaninos, estructuras formadas por uno o más residuos galoilo unidos a un núcleo de glucosa. El compuesto 6 (Rt 0.86, [M-H]331.0647) fue identificado como un monogaloil glucosa (Figura 1) por su $[\mathrm{M}-\mathrm{H}]-\mathrm{y}$ la presencia de fragmentos correspondientes a la pérdida del residuo de azúcar, para dar la $[\mathrm{M}-\mathrm{H}]$ - del ácido gálico (m/z 169), seguida de la pérdida de un grupo $\mathrm{CO}_{2}$ (m/z 125). El compuesto 34 (Rt 5.69, $[\mathrm{M}-\mathrm{H}]-787.0983)$ se identificó como un derivado tetragaloil glucosa con fragmentos característicos de la pérdida de residuos galoilo (152) y galoilo más agua (170), así como la presencia del ion con m/z 169 (Boulekbache-Makhlouf et al., 2010). También el compuesto 28 (trigaloil glucosa) se identificó como galotanino (trigaloil glucosa, Rt 5.23, [M-H]635.0878) aunque no se obtuvo su patrón de fragmentación. Otros autores han encontrado galotaninos con una y dos unidades de ácido gálico (Alvarez-Parrilla, Urrea-López \& de la Rosa, 2018) pero el presente es el primer reporte de las formas tri y tetra galoiladas. Los galotaninos fueron menos abundantes y diversos que los elagitaninos, que serán descritos a continuación.

Se encontraron 8 derivados glicosilados (donde el carbohidrato puede ser hexosa o pentosa, no necesariamente glucosa), metilados o galoilados del ácido elágico, o combinaciones entre ellos; aunque la identidad de los compuestos 30, 35, y 41 no pudo ser del todo confirmada por su patrón de fragmentación. Los compuestos 30 (Rt 5.46, [M-H]- 463.0505) y 35 (Rt 6.04, $[\mathrm{M}-\mathrm{H}]-433.0392)$ fueron identificados tentativamente como un hexósido y pentósido de ácido elágico, respectivamente; ambos han sido descritos en nuez pecana (Gong \& Pegg, 2017; Robbins, Ma, Wells, Greenspan \& Pegg, 2014) sin embargo, su fragmento característico tiene una $\mathrm{m} / \mathrm{z}$ de 301 , correspondiente a la pérdida de la hexosa (162) o pentosa (132). En el presente trabajo el fragmento liberado (m/z 300) correspondería a la pérdida del carbohidrato seguido de un átomo de hidrógeno adicional (163 y 133, respectivamente). Otros trabajos han identificado un fragmento similar $(\mathrm{m} / \mathrm{z}$ 300) como producto de hexósidos y pentósidos de ácido elágico (Sandhu \& Gu, 2010); sin embargo, esta información no es suficiente para confirmar la identidad, ya que en nuestros resultados no se observó la presencia de fragmentos característicos del ácido elágico, como son los de m/z 257 y 229 (Mullen, Yokota, Lean \& Crozier, 2003). De manera similar, el compuesto 41 (Rt 7.3, [M-H]- 315.0128) puede ser identificado tentativamente como metil-ácido elágico, también descrito previamente en nuez pecana (de la Rosa, ÁlvarezParrilla \& Shahidi, 2011), y el fragmento de m/z 300 (299.989 masa exacta), correspondiente a la pérdida del grupo metilo (15), puede considerarse un buen indicador de la identidad del compuesto. Sin embargo, de nuevo, no existen fragmentos característicos del ácido elágico y no se encontraron reportes 
del patrón de fragmentación del metil ácido elágico, por lo que consideramos que no se cuenta con información suficiente para confirmar su identificación. Por otro lado, el compuesto 37 (Rt 6.27) presentó una [M-H]- de 300.9961 y fragmentos característicos del ácido elágico (incluyendo los de m/z 257 y 229), por lo que pudo ser identificado con certeza. Los compuestos 38 (Rt 6.84, [M-H]- 477.0656) y 40 (Rt 7.07, $[\mathrm{M}-\mathrm{H}]-447.0551)$ fueron identificados como derivados glicosilados del metil ácido elágico, hexósido y pentósido, respectivamente. Su identidad pudo ser confirmada por la presencia, en ambos casos, de fragmentos correspondientes a la pérdida del carbohidrato $(\mathrm{m} / \mathrm{z} 315)$ y a la pérdida del carbohidrato y el grupo metilo (m/z 300). Los compuestos 39 (Rt 6.96, [M-H]- 585.0503) y 42 (Rt 7.54, [M-H]- 599.0656) fueron identificados como galoil pentósidos de ácido elágico y de metil ácido elágico, respectivamente. En ambos casos se observaron fragmentos correspondientes a la pérdida del residuo galoil-pentosa (284), para quedar $\mathrm{m} / \mathrm{z}$ de 301 para el compuesto 39 y el 315 para el compuesto 42, coincidiendo con lo reportado en la literatura por (Gong \& Pegg, 2017; Robbins, Ma, Wells, Greenspan \& Pegg, 2014). También se observó en ambos la liberación de ácido gálico (fragmentos con $\mathrm{m} / \mathrm{z}$ 169), en el compuesto 42 la pérdida de un residuo galoilo (fragmento con $\mathrm{m} / \mathrm{z} 447$ ) y en el compuesto 39 la de un residuo galoilo con una molécula de agua (fragmento con $\mathrm{m} / \mathrm{z} 415)$. Todos los derivados de ácido elágico encontrados en el presente trabajo ya han sido reportados en nuez pecana (Álvarez-Parrilla, Urrea-López \& de la Rosa, 2018).

Finalmente, se encontraron 6 elagitaninos, cuya característica principal es la presencia de grupos HHDP. Cuando el HHDP es liberado por hidrólisis se lactoniza para dar lugar al ácido elágico, que puede ser cuantificado como producto de la hidrólisis de elagitaninos (Gong \& Pegg, 2017; Lee, Johnson \& Talcott, 2005). Los compuestos 14 y 17 (Rt 2.01 y 2.93, [M-H]- 783.068) fueron identificados como isómeros de diHHDP-hexosa, probablemente pedunculagina, de acuerdo a su patrón de fragmentación (Boulekbache-Makhlouf et al., 2010). En ambos se observaron los mismos fragmentos: el de m/z 301 (300.996) que corresponde a la liberación de ácido elágico por la pérdida de un grupo HHDP-glucosa (482) y el de $\mathrm{m} / \mathrm{z} 481$ que corresponde a la pérdida de un grupo HHD qu P (302). También se observó un fragmento de $\mathrm{m} / \mathrm{z} 275$, sin embargo no se encontró el que corresponde a la pérdida de una molécula de agua, característico de elagitaninos con enlace C-glicosídico en los que la molécula de glucosa se encuentra en conformación abierta (Boulekbache-Makhlouf et al., 2010; Barry, Davies \& Mohamed, 2001), por lo que se considera probable que estos compuestos correspondan a dos anómeros de la pedunculagina $u$ otras formas de di-HHDP-hexosa con enlace O-glicosídico. No existen reportes previos de este compuesto en extractos de nuez pecana. El compuesto 21, (Rt 3.85, [M-H]- 633.072) correspondió al galoil-HHDP-glucosa, que había sido identificado por Gong \& Pegg (2017) en nuez pecana, por la presencia de fragmentos correspondientes a la pérdida del residuo galoilo más agua (fragmento con $\mathrm{m} / \mathrm{z} 463$ ) y a la liberación de ácido elágico (fragmento con $\mathrm{m} / \mathrm{z} 301$ [masa exacta de 300.996]); en el presente análisis también se pudo observar un fragmento correspondiente a la liberación de ácido gálico (m/z 169). Cabe mencionar que se identificaron por su masa exacta y distribución isotópica otros dos isómeros de galoil-HHDP-glucosa (compuestos 7, Rt 0.98 y 12, Rt 1.67).

El compuesto 22 (Rt 4.2) presentó una [M-H]- de 935.0793 con un fragmento de $\mathrm{m} / \mathrm{z} 633$ correspondiente a la pérdida de un residuo HHDP-glucosa, así como uno de $\mathrm{m} / \mathrm{z} 783$ por la pérdida de un residuo galoilo. Se presentaron también otros fragmentos (m/z 275 y m/z 169, similares a los encontrados en galotaninos antes descritos) pero ninguno correspondiente a la pérdida de una molécula de agua, lo que sugiere que este compuesto es un isómero de casuarictina, es decir un galoil-di-HDDP-glucosa con conformación cerrada de la glucosa (Boulekbache-Makhlouf et al., 2010; Barry, Davies \& Mohamed, 2001). Este compuesto mostró un isómero con Rt de 3.39 (compuesto 19, Tabla I). El compuesto 26 (Rt 4.89) mostró una [M-H]- de 933.0639, dos unidades menor que el compuesto 22 (galoil-di-HDDP-glucosa), lo que indica la formación de un enlace por oxidación entre el grupo galoil y un grupo HHDP adyacente (Barry, Davies \& Mohamed, 2001). Este tipo de estructura puede corresponder a elagitaninos como castalagina/vescalagina o praecoxina-D, aunque el patrón de fragmentación y la literatura disponible no permiten distinguirlo con certeza. Sin embargo, si es posible aseverar que se trata de alguno de estos taninos, ya que muestra fragmentos como el de m/z 451 (450.99) que corresponde a la liberación de un residuo trigaloilo y el de m/z 301 (300.996) correspondiente a la liberación de ácido elágico; estos fragmentos han sido descritos por Hager, Howard, Liyanage, Lay \& Prior, (2008), quien identificó castalagina/vescalagina mediante HPLC-MS/MS en extractos de zarzamoras (Rubus $s p$.), y por Grace, Warlick, Neff \& Lila, (2014), quien aisló y caracterizó la fragmentación de praecoxina $\mathrm{D}$ a partir de extractos de nuez de Castilla (Juglans regia). Por último, el compuesto 33 (Rt 5.6-5.69, [M-H]- 1103.087) se identificó como sanguina H-2 de acuerdo a la comparación de su patrón de fragmentación con el descrito por Kool, Comeskey, Cooney \& McGhie, (2010), quien aisló y caracterizó a este compuesto a partir de extractos de zarzamora de la especie Rubus loganbaccus x baileyanus, observando fragmentos de $\mathrm{m} / \mathrm{z} 1059,935$ y 633, los mismos que fueron detectados en el presente estudio. Una búsqueda bibliográfica exhaustiva nos indicó que este es el primer estudio que reporta la presencia de elagitaninos como casuarictina, castalagina/vescalagina, praecoxina D o sanguina H-2 en nuez pecana. Los compuestos más abundantes en el extracto acetónico de nuez pecana fueron el pentósido de ácido elágico (solo identificación tentativa), el dímero de procianidina B con Rt 4.54 y el galoil- 
HHDP-glucosa con Rt 3.85. En el extracto etanólico también fue más abundante el pentósido de ácido elágico, seguido por monogaloil glucosa y catequina libre.

\section{Conclusiones}

Este estudio permitió demostrar que el perfil polifenólico de la almendra y nuez pecana es claramente diferente, dominando en la primera flavonoides y en la segunda taninos condensados e hidrolizables (sobre todo elagitaninos). Se describió por primera vez la presencia de elagitaninos como casuarictina, castalagina/vescalagina, praecoxina D o sanguina H-2 en nuez pecana. Por otro lado, se demostró también que los extractos etanólicos muestran una riqueza, en cantidad y diversidad, de CF similar al de los extractos acetónicos.

\section{Agradecimientos}

Los autores agradecen a CONACYT por el financiamiento otorgado al proyecto CB-2016-286449, así como a la UACJ $\mathrm{y}$ al CIATEJ por las facilidades otorgadas para la realización de una estancia sabática de Laura A. de la Rosa y Emilio Álvarez-Parrilla. A Víctor M. Alcantar Rosales del CIATEJ por su valioso apoyo para realizar el análisis de las muestras en el HPLC-QTOF. A Jasmín C. Stevens Barrón y Gaspar A. Torres Aguirre por su apoyo en la obtención de los extractos.

\section{REFERENCIAS}

Abu-Reidah, I.M., (2013). Characterization of phenolic compounds in highly-consumed vegetable matrice by using advanced analytical techniques (Caracterización de compuestos fenólicos en matrices vegetales mediante técnicas analíticas avanzadas). Doctoral thesis, Granada: Editorial de la Universidad de Granada.

Abu-Reidah, I. M., Ali-Shtayeh, M. S., Jamous, R. M., Arráez-Román, D. \& Antonio Segura-Carretero, A. (2015). HPLC-DAD-ESI-MS/MS screening of bioactive components from Rhus coriaria L. (Sumac) fruits. Food Chem., 166, 179-191. DOI: 10.1016/j. foodchem.2014.06.011

Alasalvar, C. \& Shahidi, F. (2008). Tree nuts: Composition, phytochemicals, and health effects. Boca Raton: CRC Press.

Álvarez-Parrilla, E., Urrea-López, R. \& de la Rosa, L. A. (2018). Bioactive components and health effects of pecan nuts and their by-products: a review. J. Food Bioact., 1, 56-92. DOI: $10.31665 / J F B .2018 .1127$

Aune, D., Keum, N., Giovannucci, E., Fadnes, L. T., Boffetta, P., Greenwood, D. C., Tonstad, S., Vatten, L. J., Riboli, E. \& Norat, T. (2016). Nut consumption and risk of cardiovascular disease, total cancer, all-cause and cause specific mortality: a systematic review and dose-response meta-analysis of prospective studies. BMC Medicine, 14:207, 1-14. DOI: 10.1186/s12916-016-0730-3

Barry, K. M., Davies, N. W. \& Mohamed, C. L. (2001). Identification of hydrolysable tannins in the reaction zone of Eucalyptus nitens wood by High Performance Liquid Chromatography-Electrospray Ionisation Mass Spectrometry. Phytochem. Anal., 12, 120-127. DOI: 10.1002/pca.548

Boulekbache-Makhlouf, L., Meudec, E., Chibane, M., Mazaruic, J. -P., Slimani, S., Henry, M., Cheynier, V. \& Madani, K. (2010). Analysis by High-Performance Liquid Chromatography Diode Array Detection Mass Spectrometry of phenolic compounds in fruit of Eucalyptus globulus cultivated in Algeria. J. Agric. Food Chem,. 58, 12615-12624. DOI: 10.1021/jf1029509

Chang, S. K., Alasalvar, C., Bolling, B. W. \& Shahidi, F. (2016). Nuts and their co-products: The impact of

processing (roasting) on phenolics, bioavailability, and health benefits - A comprehensive review. J. Funct. Foods, 26, 88-122. DOI: 10.1016/j.jff.2016.06.029

de la Rosa, L. A., Álvarez-Parrilla, E. \& Shahidi, F. (2011) Phenolic compounds and antioxidant activity of kernels and shells of mexican pecan (Carya illinoinensis). $J$. Agric. Food Chem., 59, 152-162. DOI: 10.1021/jf1034306

Fabre, N., Rustan, I., Hoffmann, E. \& Quetin-Leclercq, J. (2001). Determination of flavone, flavonol, and flavanone aglycones by negative ion liquid chromatography electrospray ion trap mass spectrometry. J. Am. Soc. Mass Spectrom., 12, 707-715. DOI: 10.1016/S10440305(01)00226-4

Gong, Y. \& Pegg, R. B. (2017). Separation of ellagitannin-rich phenolics from U.S. pecans and chinese hickory nuts using Fused-Core HPLC columns and their characterization. $J$. Agric. Food Chem., 65, 5810-5820. DOI: 10.1021/acs. jafc. $7 \mathrm{~b} 01597$

Grace, M. H., Warlick, C. C., Neff, S. A. \& Lila, M. A. (2014). Efficient preparative isolation and identification of walnut bioactive components using high-speed counter-current chromatography and LC-ESI-IT-TOF-MS. Food Chem., 158, 229-238. DOI: 10.1016/j.foodchem.2014.02.117

Hager, T. J., Howard, L. R., Liyanage, R., Lay, J. O. \& Prior, R. L. (2008). Ellagitannin composition of blackberry as determined by HPLC-ESI-MS and MALDI-TOFMS. J. Agric. Food Chem., 56, 661-669. DOI: 10.1021/ jf071990b

Justesen, U. (2000). Negative atmospheric pressure chemical ionisation low-energy collision activation mass spectrometry for the characterisation of flavonoids in extracts of fresh herbs. J. Chromatogr. A., 902, 369-379. DOI: 10.1016/S0021-9673(00)00861-X

Kool, M. M., Comeskey, D. J., Cooney, J. M. \& McGhie, T. K. (2010). Structural identification of the main ellagitannins of a boysenberry (Rubus loganbaccus x baileyanus Britt.) extract by LC-ESI-MS/MS, MALDI-TOF-MS and NMR spectroscopy. Food Chem., 119, 1535-1543. DOI: 10.1016/j.foodchem.2009.09.039

Lamuel-Raventos, R. M. \& St. Onge, M. -P. (2017). Prebiotic nut compounds and human microbiota. 
Crit. Rev. Food Sci. Nutr., 57, 3154-3163. DOI: 10.1080/10408398.2015.1096763

Lee, J. - H., Johnson, O. V. \& Talcott, S. T. (2005). Identification of ellagic acid conjugates and other polyphenolics in muscadine grapes by HPLC-ESI-MS. J. Agric. Food Chem., 53, 6003-6010. DOI: 10.1021/jf050468r

Monagas, M., Garrido, I., Lebrón-Aguilar, R., Bartolomé, B. \& Gómez-Cordovés, C. (2007). Almond (Prunus dulcis (Mill.) D.A. Webb) skins as a potential source of bioactive polyphenols. J. Agric. Food Chem., 55, 8498-8507. DOI: 10.1021/jf071780z

Moqbel, H., Hawary, S. S. E. D. E., Sokkar, N. M., ElNaggar, E. M. B., Boghdady, N. E. \& Halawany, A. M. E. (2018). HPLC-ESI-MS/MS characterization of phenolics in Prunus amygdalus, cultivar "umm alfahm" and its antioxidant and hepatoprotective activity. J. Food Meas. Charact., 12, 808-819. DOI: 10.1007/s11694-017-9695-

Motilva, M. -J., Serra, A. \& Macià, A. (2013). Analysis of food polyphenols by ultra high-performance liquid chromatography coupled to mass spectrometry: An overview. J. Chromatogr. A., 1292, 66-82. DOI: 10.1016/j. chroma.2013.01.012

Mullen, W., Yokota, T., Lean, M. E. J. \& Crozier, A. (2003). Analysis of ellagitannins and conjugates of ellagic acid and quercetin in raspberry fruits by $\mathrm{LC}-\mathrm{MS}^{\mathrm{n}}$. Phytochem., 64, 617-624. DOI: 10.1016/S0031-9422(03)00281-4
Robbins, K. S., Ma, Y., Wells, M. L., Greenspan, P. \& Pegg, R. B. (2014). Separation and characterization of phenolic compounds from U.S. pecans by Liquid ChromatographyTandem Mass Spectrometry. J. Agric. Food Chem., 62, 4332-4341. DOI: 10.1021/jf500909h

Sandhu, A. K. \& Gu, L. (2010). Antioxidant capacity, phenolic content, and profiling of phenolic compounds in the seeds, skin, and pulp of Vitis rotundifolia (Muscadine grapes) as determined by HPLC-DAD-ESI-MS ${ }^{\mathrm{n}}$. J. Agric. Food Chem. 58, 4681-4692. DOI: 10.1021/jf904211q

Sauceda, A. E. Q., Sáyago-Ayerdi, S. G., Ayala-Zavala, J. F., Wall-Medrano, A., de la Rosa, L. A., GonzálezAguilar, G. A. \& Álvarez-Parrilla, E. (2018). Biological Actions of Phenolic Compounds. En: Yahia, E. M. (Ed.) Fruit and Vegetable Phytochemicals: Chemistry and Human Health (pp. 286-307). Hoboken: John Wiley and Sons Ltd.

Torres-Aguirre, G. A., Muñoz-Bernal, O. A., Álvarez-Parrilla, E., Núñez-Gastélum, J. A., Wall-Medrano, A., SáyagoAyerdi, S. G. \& de la Rosa, L. A. (2018). Optimización de la extracción e identificación de compuestos polifenólicos en anís (Pimpinella anisum), clavo (Syzygium aromaticum) y cilantro (Coriandrum sativum) mediante HPLC acoplado a espectrometría de masas. TIP Revista Especializada en Ciencias Químico-Biológicas, 21, 103 115. DOI: $10.22201 /$ fesz.23958723e.2018.2.4 